\title{
Impact loading of ductile rectangular plates
}

\author{
N. Jones \\ Impact Research Centre, Department of Engineering, \\ University of Liverpool, $U K$
}

\begin{abstract}
In many industries, rigid-plastic methods of analysis are a useful design aid for safety calculations, hazard assessments and forensic investigations of ductile structures, which are subjected to large dynamic loads producing an inelastic rsponse. This paper examines the behaviour of a rectangular plate struck at the centre by a rigid mass impact loading. A theoretical method has been developed previously which retains the influence of finite transverse displacements, or geometry changes. It is used in this paper to predict the maximum permanent transverse displacements of plates having boundary conditions characterised by a resisting moment $\mathrm{mM}_{0}$ around the entire boundary, where $\mathrm{m}=0$ and $\mathrm{m}=1$ give the two extreme cases of simply supported and fully clamped supports, respectively.

The theoretical predictions are compared with some experimental data recorded on fully clamped metal rectangular plates having a range of aspect ratios and struck by masses travelling with low impact velocities up to nearly $7 \mathrm{~m} / \mathrm{s}$ and which produce large ductile deformations without failure. The theoretical analysis gives reasonable agreement with the corresponding experimental data for masses having blunt, conical and hemisperical impact faces.

For sufficiently large initial impact energies, the projectile would perforate a plate and, for completeness, a useful design equation is presented which predicts perforation energies larger than all of the test data, as expected.

Keywords: rectangular plate, square plate, mass impact, rigid-plastic analysis, large permanent transverse displacements, metal plate, simply supported, fully clamped, experiments, perforation.
\end{abstract}




\section{Introduction}

Simple and reliable theoretical methods are still valuable for design purposes, particularly for preliminary design and hazard assessments, and for forensic investigations after accidents. A theoretical rigid-plastic method was developed in [1] which retained the influence of large transverse displacements (i. e., geometry changes, or membrane effects) and which has been used to predict the maximum permanent transverse displacements, or damage, of rectangular plates when subjected to a pressure pulse causing plastic strains. It was shown how this method can be simplified with an approximate yield condition which allows useful design equations to be obtained. This method was also used to examine the impulsive, or blast, loading of rectangular plates, and good agreement was found with experimental results recorded on ductile metal plates having various aspect ratios [2-4]. The method was extended to obtain the behaviour of circular plates [4-6] and square plates [6] struck by a solid mass at the centre, and again good agreement was reported with the maximum permanent transverse displacements observed in experimental tests on ductile metal plates.

This paper extends the above theoretical method to obtain the maximum permanent transverse displacements, or ductile damage, for a rectangular plate struck by a solid mass at the centre. It turns out that a relatively simple equation was obtained which gives good agreement with experimental data recorded on ductile metal rectangular plates having a range of aspect ratios from 0.4 to 1 and reported in [7].

Thus, design equations are now available for predicting the maximum permanent transverse displacements, or damage, for circular plates and rectangular plates (including square plates and beams) subjected to pressure pulses (including the limiting case of an impulsive loading) or central solid mass impacts. Moreover, these equations have been tested against experiments on ductile metal plates and are therefore suitable for design purposes, safety calculations and hazard assessments.

The next section of this paper provides a brief outline of the theoretical method which is used in section 3 to examine the behaviour of a rectangular plate subjected to a mass impact loading. Section 4 discusses briefly the experimental details of the data obtained on mild steel rectangular plates struck by a mass at the plate centre which produces large ductile deformations without any failure. Sections 5 and 6 contain a discussion and conclusions, respectively.

\section{Theoretical method for dynamic loading of plates}

A theoretical procedure was developed in [1] to provide the permanently deformed profile of an arbitrary shaped ductile plate, when subjected to large static or dynamic loads which produce plastic strains. The material is assumed to 
be rigid, perfectly plastic with a yield stress $\sigma_{o}$ and the plate has a uniform thickness $H$ and the governing equations can be simplified and written in the form

$$
\begin{aligned}
& -G \ddot{W} \dot{W}-\int_{A} \mu \ddot{w} \dot{w} d A= \\
& \int_{A}\left\{\left(M_{r}+w N_{r}\right) \dot{\kappa}_{r}+\left(M_{\theta}+w N_{\theta}\right) \dot{\kappa}_{\theta}\right\} d A \\
& +\sum_{m=1}^{n} \int_{C_{m}}\left(M_{r}+w N_{r}\right)(\partial \dot{w} / \partial r)_{m} d C_{m} \\
& +\sum_{u=1}^{v} \int_{C_{u}} Q_{r}(\dot{w}) d C_{u}
\end{aligned}
$$

where $G$ is an impact mass, and $\mu$ is the mass per unit surface area of a plate. The transverse displacement of a plate is $w$, while $\dot{w}$ and $\ddot{w}$ are the associated velocity and acceleration. $W$ is the transverse displacement of the plate immediately underneath a striking mass.

The terms on the left hand side of equation (1) are the work rate due to the inertia forces, where A is the surface area of a plate. The first term on the right hand side of equation (1) is the energy dissipated in any continuous deformation fields. The second term gives the energy dissipated in $\mathrm{n}$ plastic bending hinges, each having an angular velocity $(\partial \dot{w} / \partial r)_{m}$ across a hinge of length $C_{m}$. The final term is the plastic energy absorption in $v$ transverse shear hinges, each having a velocity discontinuity $(\dot{w})_{u}$ and a length $C_{u}$. Equation (1) ensures that the external work rate equals the internal energy dissipation.

This general equation has been used to study the dynamic plastic response of beams, and of circular, square and rectangular plates subjected to dynamic pressure pulses and also blast loadings [1-4], and for beams, circular and square plates struck at the mid-span by a rigid mass [4-6]. It is used in this paper to examine a rectangular plate struck by a rigid mass at the centre, and, since large ductile deformations are studied without any material failure, or perforation, the transverse shear term in equation (1) is not considered further. Thus, the yield condition consists of four generalised stresses which can be related by the limited interaction surface shown in Figure 2 of [6]. However, if a deformation profile consists only of rigid regions separated by plastic hinges, then the exact yield condition in Figure 1 governs plastic flow along the hinge lines. A square yield condition circumscribes the exact yield condition (maximum normal stress yield criterion), while another one which is 0.618 times as large would inscribe it, thus providing upper and lower bounds on the exact yield condition according to a corollary of the limit theorems [4]. 


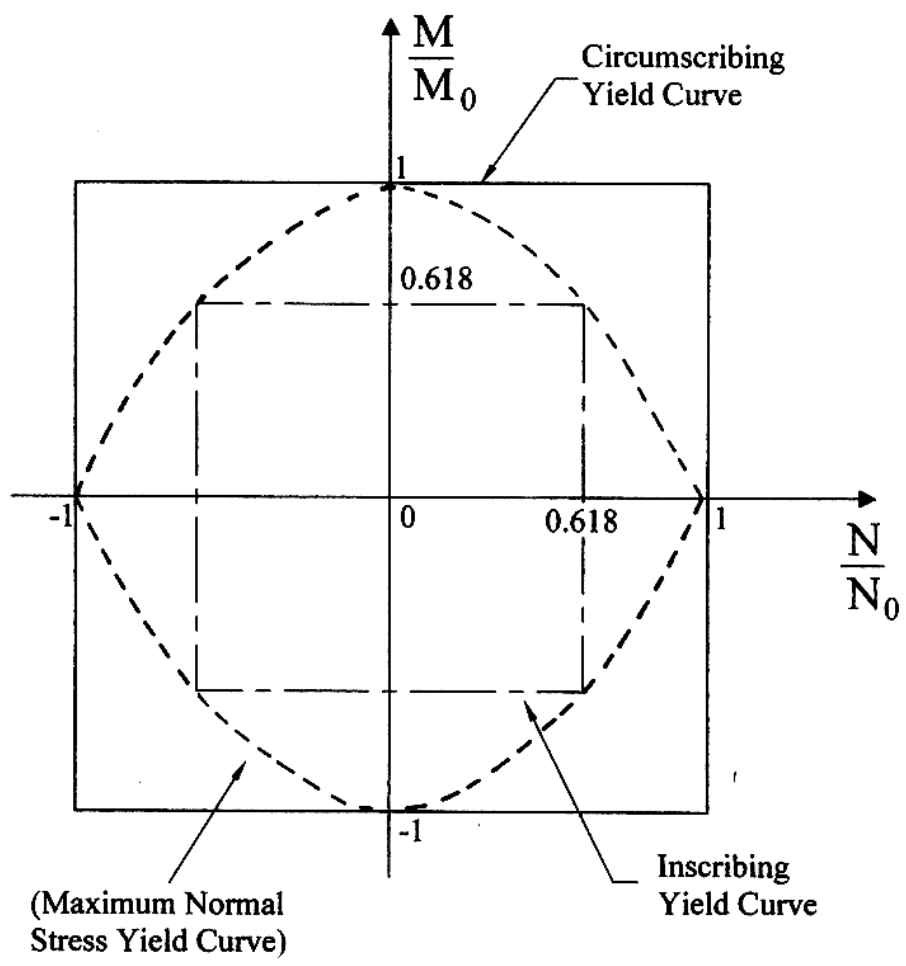

Figure 1: Yield conditions along the plastic hinge lines (including the supports) which develop within the rectangular plate in Figure 2.

\section{Mass impact loading of a rectangular plate}

The kinematically admissible transverse velocity field in Figure 2 is assumed to govern the response of the rectangular plate $(2 L x 2 B)$ in Figure 3, which has a moment resistance $m M_{o}$ around the supports $(m=0$ and $m=1$ give the simply supported and the fully clamped cases, respectively, but intermediate cases $(0<$ $m<1)$ are included in the analysis). It is assumed that the mass strikes the centre of a rectangular plate, has a negligible cross-section when compared with the plate dimensions, $\mathrm{B}$ and $\mathrm{L}$, and produces a response characterised by the transverse velocity profile in Figure 2. 


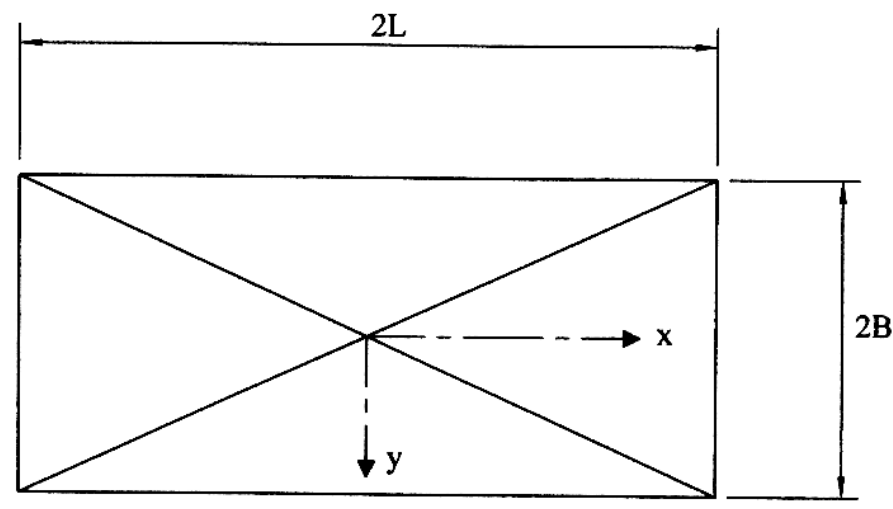

(a)

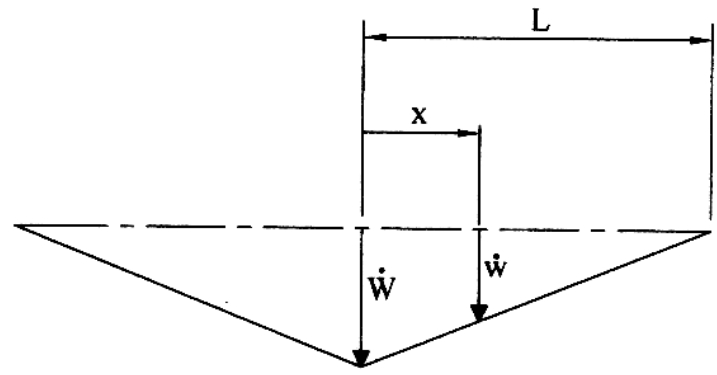

(b)

Figure 2: $\quad$ Pyramidal-shaped transverse velocity field for a rigid, perfectly plastic rectangular plate struck at the centre as shown in Figure 3. (a) plan view of plastic hinge lines which develop across diagonals and at boundaries (except for the simply supported case when $m=0$ on the four boundaries). (b) side view (in $y$ direction) of pyramidal-shaped transverse velocity field at time $t$.

Equation (1) can be written

$$
-G \ddot{W} \dot{W}-\int_{A} \mu \ddot{w} \dot{w} d A=\sum_{m=1}^{r} \int_{l_{m}}(M+N w) \dot{\theta}_{m} d l_{m}
$$




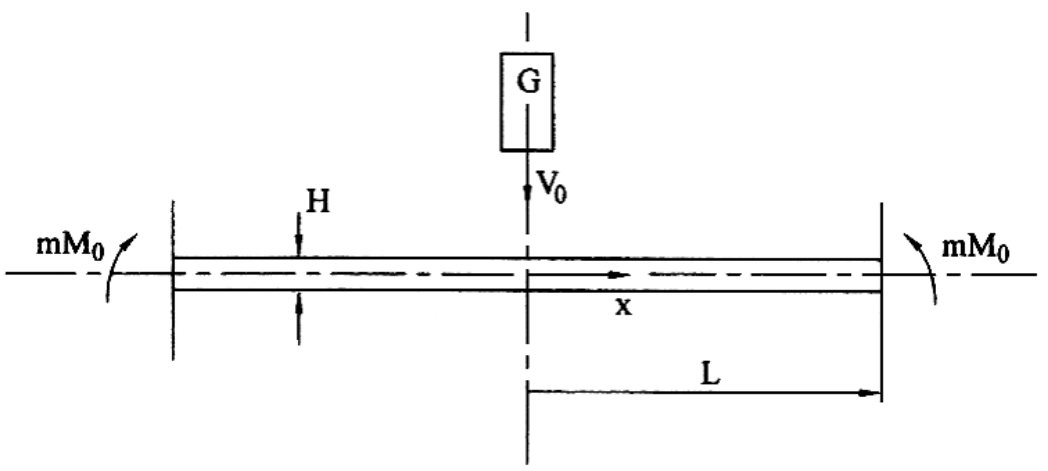

Figure 3: Rectangular plate struck at the centre by a rigid mass $G$ travelling with an impact velocity $V_{0}$. The bending resistance around the four supports is $m M_{0}$, where $0 \leq m \leq 1$ and $M_{0}$ is the plastic limit moment per unit length of the plate cross-section.

for an initially flat, rigid, perfectly plastic plate which deforms into a number of rigid regions separated by $\mathrm{r}$ stationary straight line plastic hinges, each of length $l_{m} \cdot \dot{\theta}_{m}$ is the relative angular velocity across a straight line hinge, $w$ is the transverse displacement along a hinge, and $N$ and $M$ are the membrane force and bending moment, respectively, which act on a plane which passes through a hinge and is transverse to the mid-surface of a plate.

Equation (2) for the impact problem in Figure 3, when using the transverse velocity field in Figure 2 and the square yield condition in Figure 1, yields the governing equation

$$
\ddot{W}+\alpha^{2} W=-(1+m) H \alpha^{2} / 2
$$

where $\quad \alpha^{2}=\frac{12 M_{0}\left(1+\beta^{2}\right)}{\mu H L^{2}(1+6 \gamma) \beta^{2}}, \quad \beta=\frac{B}{L} \quad$ and $\quad \gamma=\frac{G}{4 \mu B L}$

Solving equation (3) for the initial condition $w=0$ at $t=0$ and satisfying the conservation of linear momentum at $t=0$ (i.e., $\dot{W}=V_{0} /\{1+1 / 3 \gamma\}$, where $V_{0}$ is the initial velocity of the striking mass), then, for the fully clamped case with $m$ $=1$, the dimensionless maximum permanent transverse displacement is

$$
\begin{gathered}
\frac{W_{f}}{H}=\sqrt{\left\{1+\frac{2 \beta \Omega}{\left(1+\beta^{2}\right)}\right\}}-1, \\
\Omega=\frac{G V_{o}^{2}}{4 \sigma_{0} H^{3}} .
\end{gathered}
$$


Equation (5) for a square plate with $\beta=1$ reduces to the corresponding equation developed in [6]. A more complete version of this analysis, which predicts the response duration and other features, will be published in due course.

\section{Experimental details}

The plates in the theoretical analysis reported in section 3 undergo wholly ductile deformations without any material failure or perforation. Some of the data for the perforation study reported in [7] on rectangular plates were obtained for lower impact velocities than those required for perforation and gave rise to wholly ductile behaviour of a rectangular plate without failure. This data is reported in Table 1.

Table 1: Experimental data for rectangular plates struck at mid-span. $H=$ $4 \mathrm{~mm}, \sigma_{0}=262 \mathrm{MPa}, G=11.8 \mathrm{~kg}$ for blunt-tipped projectiles and $\mathrm{G}=19 \mathrm{~kg}$ for hemispherically-tipped and conically-tipped projectiles [7].

\begin{tabular}{|c|c|c|c|c|c|}
\hline shape & $\beta$ & $\begin{array}{c}2 L \times 2 B \\
\left(\mathrm{~mm}^{2}\right)\end{array}$ & $\begin{array}{c}V_{0} \\
(\mathrm{~m} / \mathrm{s})\end{array}$ & $\Omega$ & $W_{f} / H$ \\
\hline blunt & 0.4 & $100 \times 250$ & 6.49 & 7.42 & $2.10^{*}$ \\
\hline blunt & 0.5 & $100 \times 200$ & 6.49 & 7.42 & 2.03 \\
\hline blunt & 0.66 & $100 \times 150$ & 6.26 & 6.90 & 2.20 \\
\hline blunt & 0.66 & $100 \times 150$ & 6.42 & 7.25 & 1.83 \\
\hline blunt & 1 & $100 \times 100$ & 6.42 & 7.25 & 1.84 \\
\hline blunt & 1 & $200 \times 200$ & 6.57 & 7.60 & 2.12 \\
\hline hemisph'1 & 0.4 & $100 \times 250$ & 6.86 & 13.34 & $2.74^{*}$ \\
\hline hemisph'1 & 0.5 & $100 \times 200$ & 6.72 & 12.79 & 2.66 \\
\hline hemisph'1 & 0.66 & $100 \times 150$ & 6.57 & 12.23 & 2.51 \\
\hline hemisph'1 & 1 & $100 \times 100$ & 6.42 & 11.67 & 2.36 \\
\hline hemisph'1 & 1 & $200 \times 200$ & 6.86 & 13.32 & 2.91 \\
\hline conical & 0.5 & $100 \times 200$ & 6.57 & 12.23 & $2.51^{*}$ \\
\hline conical & 0.66 & $100 \times 150$ & 6.64 & 12.51 & $2.40^{*}$ \\
\hline conical & 1 & $100 \times 100$ & 5.60 & 8.89 & 2.01 \\
\hline conical & 1 & $200 \times 200$ & 6.64 & 12.51 & $2.76^{*}$ \\
\hline
\end{tabular}

*Indicates that the plating has cracked underneath the projectile impact location.

The uniaxial tensile characteristics of the $H=4 \mathrm{~mm}$ thick mild steel plate are $\sigma_{0}=262 \mathrm{MPa}, \sigma_{u}=359 \mathrm{MPa}$ and $\varepsilon_{f}=37.5 \%$. The projectiles have a 10.16 mm diameter $(d)$ body, and the impact faces are blunt, hemispherical or conical $\left(90^{\circ}\right.$ included angle). A drop hammer rig was used for the tests and the impact mass $(G)$ was $11.8 \mathrm{~kg}$ for the blunt-tipped projectiles and $19 \mathrm{~kg}$ for the hemispherically-tipped and conically-tipped projectiles. The initial impact 
velocity $\left(V_{0}\right)$ was recorded using a laser Doppler velocimeter [8] and the maximum permanent transverse displacements were measured at the plate centre. Further details of the test arrangement is reported in $[7,9,10]$.

\section{Discussion}

It is evident from equation (5) that the theoretical predictions for $W_{f} / H$ collapse on to a single curve irrespective of the plate aspect ratio $\beta$ when $2 \beta \Omega /\left(1+\beta^{2}\right)$ is used in the abscissa, as shown in Figure 4. A yield stress $0.618 \sigma_{0}$ inscribes the exact yield condition in Figure 1 and gives rise to the "upper bound" predictions according to equation (5) in Figure 4. The experimental data in Table 1 is also plotted in Figure 4 for values of $0.4 \leq \beta \leq 1$ and for three shapes of the projectile impact faces. First of all, it is evident that most of the

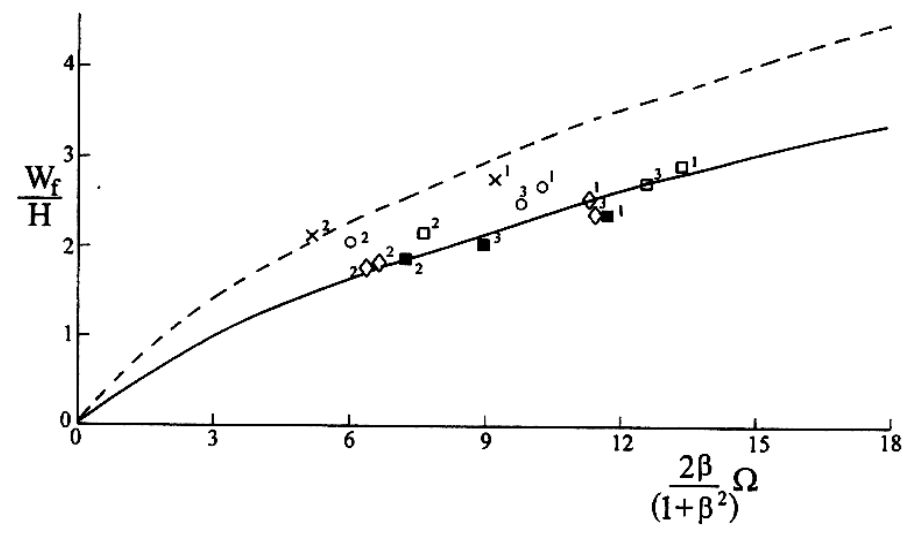

: equation (5), circumscribing yield condition

------ : equation (5), inscribing yield condition

experimental data from Table 1:

$\mathrm{X}, \circ, \diamond ; \beta=0.4,0.5,0.66$, respectively.

$\square, \mathbf{\square} ; \beta=1$ for $200 \mathrm{~mm} \times 200 \mathrm{~mm}$ and $100 \times 100 \mathrm{~mm}$ plates, respectively.

superscripts 1, 2 and 3 denote projectiles with hemispherical, blunt and conical ( $90^{\circ}$ included angle) impact faces, respectively.

Figure 4: Maximum dimensionless permanent transverse displacements at the centre of fully clamped rectangular plates versus the dimensionless initial kinetic energy. 
experimental data is bounded by the "upper" and "lower" bound predictions of equation (5). Secondly, a trend is discerned which indicates that the experimental data migrates from the "lower bound" curve to the "upper bound" curve as $\beta$ decreases for both the hemispherically-tipped and blunt-tipped projectiles. However, it should be noted that both of the $\beta=0.4$ plates had some cracking underneath the impact site. The experimental results for the conical projectiles are not distinct from those for the two other projectile nose shapes and remained fairly close to the "lower bound" predictions of equation (5); all of these plates, except one, had cracked underneath the projectile impact location.

The rectangular plates in [7] are made from mild steel which usually is a strain rate sensitive material, although the dynamic material characteristics are not available for this particular plate material. The dynamic stress state in a rectangular plate is complex and is not uniaxial. In this regard, it is interesting to observe in Figure 11 of [11] that the maximum permanent transverse deflections of impulsively loaded rectangular plates made from aluminium alloy 6061T6, which is an essentially strain rate insensitive material, and mild steel, differ by about $10-15 \%$, whereas the difference would be nearer $30-40 \%$ for a similar comparison on beams [3, 12] which have a less complex stress state. Nevertheless, if strain rate effects were incorporated into equation (5), as shown in [4] for blast loaded plates, then the theoretical curves in Figure 4 would be lower, although the strain rate effects for the low impact velocities in Table 1 are not expected to be large as those in [11] for blast loadings. It should be remarked that large plastic strains cause a reduction in the material strain rate effect [13], when compared with relatively small strain problems.

The experimental study reported in [7] has examined the threshold conditions for the perforation of plates which occurs at impact energies larger than those associated with the data for the wholly ductile deformations in Table 1. A perforation equation

$$
\Omega_{p}=(\pi / 4)(d / H)+(d / H)^{1.53}(S / d)^{0.21}
$$

was presented at the 1992 SUSI meeting [14] for circular plates, struck by cylindrical projectiles having blunt impact faces and travelling at low velocities. It turns out that this equation gave good estimates for the dimensionless perforation energy, $\Omega_{p}$, of rectangular mild steel plates when the span, $S$, is taken as the plate breadth $2 B$.

Equation (7) for $2 B=100 \mathrm{~mm}$ predicts $\Omega_{p}=8.72$, while $2 B=200 \mathrm{~mm}$ gives $\Omega_{p}=9.77$. These values are larger than the experimental results for the square plates struck by blunt-tipped projectiles in Table 1 and Figure 4 (identified with a superscript 2) since $2 \beta \Omega_{p} /\left(1+\beta^{2}\right)=\Omega_{p}$ for $\beta=1$. The factor $2 \beta \Omega /\left(1+\beta^{2}\right)$ in equation (5), which is used for the abscissa in Figure 4, is $0.69 \Omega, 0.80 \Omega$ and $0.92 \Omega$ for $\beta=0.4,0.5$ and 0.66 , respectively, and yields the corresponding values of $2 \beta \Omega_{p} /\left(1+\beta^{2}\right)=6.01,6.97$ and 8.01. As anticipated, these values lie 
above all of the corresponding rectangular plate data struck by blunt-tipped projectiles, which are identified with a superscript 2 in Figure 4. Moreover, the experimental results in $[7,9]$ reveal that the threshold values of the dimensionless perforation energy, $\Omega_{p}$, are higher for projectiles having hemispherical and conical impact faces in this series of experimental tests.

\section{Conclusions}

A theoretical rigid, perfectly plastic method, which has been developed previously for initially flat plates having an arbitrary shaped boundary and subjected to either static or dynamic loadings which produce large plastic strains and finite transverse displacements, has been used to study the behaviour of a rectangular plate struck by a mass at the centre. A relatively simple equation has been derived for the maximum permanent transverse displacements which can be used for preliminary design purposes and accident investigations. The theoretical predictions have been developed for a plate having a resisting moment $\mathrm{mM}_{0}, 0<$ $\mathrm{m}<1$, on the entire boundary. In the particular case of $\mathrm{m}=1$ for the fully clamped case, the theoretical predictions were compared with some experimental data recorded on fully clamped rectangular plates having aspect ratios between 0.4 and 1 (square) and struck transversely by masses with cylindrical bodies travelling up to about $7 \mathrm{~m} / \mathrm{s}$. The theoretical predictions provide a reasonable estimate of the corresponding experimental data.

For completeness, an empirical equation has been used to predict the initial impact energy required to perforate a plate by a blunt-faced missile. This equation predicts perforation energies which lie above all of the corresponding experimental data for large-mass and low-velocity impacts on steel plates.

\section{Acknowledgements}

The author is grateful to Mrs J. Jones for her secretarial assistance and to Mrs I. Arnot for her assistance with the figures.

\section{References}

[1] Jones, N., A Theoretical Study of the Dynamic Plastic Behavior of Beams and Plates with Finite-Deflections, International Journal of Solids and Structures, 7, pp. 1007-1029, 1971.

[2] Jones, N., Baeder, R. A., An Experimental Study of the Dynamic Plastic Behavior of Rectangular Plates, Symposium on Plastic Analysis of Structures, 1, pp. 476-497, pub. by Ministry of Education, Polytechnic Institute of Jassy, Civil Eng. Faculty, 1972.

[3] Jones, N., A Literature Review of the Dynamic Plastic Response of Structures, The Shock and Vibration Digest, 7(8), pp. 89-105, 1975. 
[4] Jones, N., Structural Impact, Cambridge University Press, pp. 575, 1989, Paperback edition, 1997. Chinese edition translated by Ping Jiang and Lili Wang, Sichuan Education Press, Chengdu, 1994

[5] Jones, N., Kim, S-B., Li, Q. M., Response and Failure Analysis of Ductile Circular Plates Struck by a Mass, Trans. ASME, Journal of Pressure Vessel Technology, 119(3), pp. 332-342, 1997.

[6] Jones, N., On the Mass Impact Loading of Ductile Plates, Defence Science Journal, Defence Research and Development Organisation, India, 53(1), pp. 15-24, 2003.

[7] Jones, N., Birch, R. S., Duan, R., Low Velocity Perforation of Mild Steel Rectangular Plates with Projectiles having Different Shaped Impact Faces, ASME, Journal of Pressure Vessel Technology, 130(3), pp. 031206-1 to 031206-8, August 2008.

[8] Birch, R. S., Jones, N., Measurement of Impact Loads Using a Laser Doppler Velocimeter, Proc. I. Mech. E., 204(C1), pp. 1-8, 1990.

[9] Jones, N., Birch, R. S., Low Velocity Perforation of Mild Steel Circular Plates with Projectiles having Different Shaped Impact Faces, ASME, Journal of Pressure Vessel Technology, 130(3), pp 031205-1 to 031205-11, August 2008.

[10] Birch, R. S., Jones, N., Jouri, W. S., Performance Assessment of an Impact Rig, proc. I. Mech. E., 202 (C4), pp. 275-285, 1988. Corrigenda 204 (C1), p.8, 1990.

[11] Jones, N., Uran, T. O., Tekin, S. A., The Dynamic Plastic Behavior of Fully Clamped Rectangular Plates, International Journal of Solids and Structures, 6, pp. 1499-1512, 1970.

[12] Jones, N., Griffin, R. N., Van Duzer, R. E., An Experimental Study Into the Dynamic Plastic Behavior of Wide Beams and Rectangular Plates, International Journal of Mechanical Sciences, 13(8), pp. 721-735, 1971.

[13] Jones, N., Some Comments on the Modelling of Material Properties for Dynamic Structural Plasticity, International Conference on the Mechanical Properties of Materials at High Rates of Strain, Oxford. Ed. J. Harding, Institute of Physics Conference Series No. 102, pp.435-445, 1989.

[14] Wen, H. M., Jones, N., Semi-Empirical Equations for the Perforation of Plates Struck by a Mass, 2nd International Conference on Structures Under Shock and Impact, Ed. P. S. Bulson, Computational Mechanics Publications, Southampton and Boston and Thomas Telford, London, pp. 369-380, 1992. 